\title{
Index of figures
}

Figure 1.1 Example time line for processing a patent family -12

Figure 1.2 The formation of a patent family -19

Figure 2.1 EPO search report header $-\mathbf{4 0}$

Figure 2.2 Contents of European search report -41

Figure 3.1 US front page showing provisional filing data (INID 60) -67

Figure 3.2 Title header of a US Statutory Invention Registration -68

Figure 4.1 Japanese publication (INID 45) and grant (INID 24) dates - $\mathbf{8 6}$

Figure 4.2 Headers for Japanese language (pre-1995 (a) and current (b)), and translated $($ PCT republications -89

Figure 4.3 Japanese national classification (INID field 52) -99

Figure 5.1 Numbers of PCT publications in each language group (1995-2018) 104

Figure 5.2 Publication sequence under the PCT system - 105

Figure 5.3 Designated states listing on PCT front page (INID 81 and 84) -107

Figure 5.4 Example record from PatentScope showing the Documents tab 113

Figure 5.5 PatentScope CLIR strategy and sample results after query translation -115

Figure 6.1 Front page data - French unexamined publication 134

Figure 6.2 Eurasian Patent Organisation - unexamined application - 172

Figure 6.3 Eurasian Patent Organisation - granted patent - 173

Figure 6.4 UK republication of a PCT application entering national phase -179

Figure 7.1 Korean unexamined and accepted front pages -225

Figure 7.2 Taiwan publication numbers under old (TW 595872-U) and new (TW I 595872-B) systems - 233

Figure 7.3 Total patent grant statistics 2015-2018 for active ASEAN states - 235

Figure 8.1 Brazilian pipeline application numbering and kind code -263

Figure 8.2 Colombian patent register contents $-\mathbf{2 8 0}$

Figure 8.3 Peruvian grant record from the PROSUR database $\mathbf{2} \mathbf{2 8 7}$

Figure 8.4 Contrasting copies of MX 351074-B - 301

Figure 9.1 South African patent strength index 2010-2015 (after

Papageorgiadis) -309

Figure 9.2 Front page of Moroccan patent validating EPO grant - $\mathbf{3 1 7}$

Figure 9.3 Front page data on Egyptian documents indicating patent term -332

Figure 9.4 Sample announcement in Kenya Gazette of GB re-registration — 338

Figure 9.5 Home page of ARIPO Regional IP Database - 365

Figure 9.6 OAPI Bulletin entry and link (“Consulter le mémoire") to corresponding specification -367

Figure 9.7 Gulf Cooperation Council Patent Office - old style gazette entry - $\mathbf{3 7 0}$

Figure 9.8 Gulf Cooperation Council Patent Office - new style gazette entry (two parts) -371

Figure 10.1 Chinese industry support centres (website map) - $\mathbf{3 8 3}$

Figure 10.2 The South Korea Regional IP Centers network (website map) - 384

Figure 11.1 MIMOSA application searching ESPACE-ACCESS disks and linking to other servers - 395

Figure 11.2 An example of a Markush (generic) chemical structure - 428

Figure 12.1 The UNESCO Libraries Portal -465 
Figure 16.1 WON directory of public register sources $-\mathbf{5 1 0}$

Figure 16.2 Continuity Data tab from Public PAIR - $\mathbf{5 1 2}$

Figure 16.3 Example record (truncated) from EPO Register - 515

Figure 16.4 EPO file inspection - table of contents - $\mathbf{5 1 6}$

Figure 16.5 Global Dossier record via the USPTO (WO 2011/129660) - 528

Figure 18.1 Family equivalents classified to different IPC levels $-\mathbf{5 4 5}$ 